\title{
A Homolog of the Arabidopsis TIME FOR COFFEE Gene Is Involved in Nonhost Resistance to Wheat Stem Rust in Brachypodium distachyon
}

\author{
Rafael Della Coletta, ${ }^{1,2}$ Anastasiya A. Lavell, ${ }^{1}$ and David F. Garvin ${ }^{1,3+}$ \\ ${ }^{1}$ Department of Agronomy and Plant Genetics, University of Minnesota, St. Paul, MN 55108, U.S.A. \\ ${ }^{2}$ CAPES Foundation, Ministry of Education of Brazil, Brasilia, DF, Brazil \\ ${ }^{3}$ Plant Science Research Unit, United States Department of Agriculture-Agricultural Research Service, St. Paul, MN 55108, \\ U.S.A.
}

Accepted 28 July 2021.

\begin{abstract}
Plants resist infection by pathogens using both preexisting barriers and inducible defense responses. Inducible responses are governed in a complex manner by various hormone signaling pathways. The relative contribution of hormone signaling pathways to nonhost resistance to pathogens is not well understood. In this study, we examined the molecular basis of disrupted nonhost resistance to the fungal species Puccinia graminis, which causes stem rust of wheat, in an induced mutant of the model grass Brachypodium distachyon. Through bioinformatic analysis, a 1-bp deletion in the mutant genotype was identified that introduces a premature stop codon in the gene Bradilg24100, which is a homolog of the Arabidopsis thaliana gene TIME FOR COFFEE (TIC). In Arabidopsis, TIC is central to the regulation of the circadian clock and plays a crucial role in jasmonate signaling by attenuating levels of the transcription factor protein MYC2, and its mutational disruption results in enhanced susceptibility to the hemibiotroph Pseudomonas syringae. Our similar finding for an obligate biotroph suggests that the biochemical role of TIC in mediating disease resistance to biotrophs is conserved in grasses, and that the correct modulation of jasmonate signaling during infection by Puccinia graminis may be essential for nonhost resistance to wheat stem rust in B. distachyon.
\end{abstract}

${ }^{\dagger}$ Corresponding author: D. F. Garvin; david.garvin@usda.gov

The use of trade, firm, or corporation names in this publication is for the information and convenience of the reader. Such use does not constitute an official endorsement or approval by the United States Department of Agriculture or the Agricultural Research Service of any product or service to the exclusion of others that may be suitable. USDA is an equal opportunity provider and employer. The complete nondiscrimination policy can be found on the USDA website.

Funding: R. Della Coletta was supported by a Coordenação de Aperfeiçoamento de Pessoal de Nível Superior fellowship (CsF- 88888.076129/ 2013-00) from the Ministry of Education of Brazil. The research was funded by United States Department of Agriculture-Agricultural Research Service appropriated projects 5062-21000-030-00D and 506221220-021-00D.

*The $e$-Xtra logo stands for "electronic extra" and indicates that a supplementary file and supplementary materials are published online.

The author(s) declare no conflict of interest.

(i) Copyright ( 2021 The Author(s). This is an open access article distributed under the CC BY 4.0 International license.
Keywords: bioinformatics, bulked segregant analysis, genomics, hormones, hormone signaling, jasmonate, mutagenesis, plant disease, plant immunity, plant responses to pathogens, proteomics and metabolomics

Plants rely on the ability to recognize conserved molecules of microorganisms in order to activate defense responses, and the plant immune system is broadly divided in two categories based on the type of molecule recognized: pathogen-associated molecular pattern (PAMP)-triggered immunity (PTI), also known as basal resistance, and effector-triggered immunity (ETI) (Jones and Dangl 2006). PTI occurs after recognition of PAMPs (elicitors), which are conserved molecules important to pathogen survival such as flagellin and chitin (Boller and Felix 2009; Dodds and Rathjen 2010), leading to defense-related gene activation (Boller and Felix 2009; Dodds and Rathjen 2010; Nürnberger and Lipka 2005). In contrast, ETI is associated with resistance $(R)$-gene-based resistance and depends on intracellular recognition of effectors produced by the pathogen attempting to suppress PTI (Boller and Felix 2009). ETI leads to stronger defense responses that usually result in hypersensitive reactions.

Each pathogen has a certain number of host species that they can infect and cause disease. This varies from a narrow host range (infecting only one host species or genus) to a broad host range (Schulze-Lefert and Panstruga 2011; Thordal-Christensen 2003). Based on this, it is possible to delineate two types of resistance: host and nonhost resistance. Host resistance occurs when some genotypes within a host species avoid infection by a specific pathogen, normally due to $R$ genes that trigger ETI and a hypersensitive response (Heath 2000; Mysore and Ryu 2004). In contrast, nonhost resistance involves complex mechanisms that result in the majority of plant species being resistant to most pathogens (Mysore and Ryu 2004). Nonhost resistance is suggested to involve both PTI and ETI (Jones and Dangl 2006; Schulze-Lefert and Panstruga 2011) but it remains a poorly understood phenomenon (Panstruga and Moscou 2020). Nonetheless, nonhost resistance is effective because it employs several responses that prevent or halt pathogen growth, including preformed barriers such as wax layers, plant cell walls, and secondary metabolites (Mysore and Ryu 2004; Nürnberger et al. 2004; Thordal-Christensen 2003). If a pathogen overcomes these obstacles, PTI may be induced, resulting in cell wall thickening, cytoskeleton reorganization, papilla formation, and production of antimicrobial compounds (Mysore and Ryu 2004; Uma et al. 2011). 
Schulze-Lefert and Panstruga (2011) proposed a model to connect nonhost resistance and pathogen host range at the molecular level, wherein both PTI and ETI contribute to nonhost resistance but their relative contributions change as the phylogenetic distance between a host and a nonhost increases. They proposed that, when genetic distance between a host and a nonhost is very large, a pathogen's effectors do not find a target to suppress PTI and, therefore, cannot infect the plant. In contrast, when host and nonhost are more closely related, it is possible that preexisting nucleotide-binding site leucine-rich repeat proteins of the nonhost may recognize common effectors of the pathogen, leading to ETI. Bettgenhaeuser et al. (2014) proposed that nonhost plant species exhibit a continuum of phenotypes to rust pathogens, ranging from no visual symptoms to the frequent presence of small pustules or a few genotypes of a plant species being susceptible. This fits with the model of Schulze-Lefert and Panstruga (2011).

Rust pathogens of the fungal genus Puccinia pose a global threat to the production of cool-season cereal crops such as wheat and barley. More than two decades ago, Ug99, a new race of stem rust caused by Puccinia graminis, emerged in Africa that overcame the resistance of gene $S r 31$, which is present in much of the wheat grown in the world and provided resistance to all stem rust races for more than 30 years (Ellis et al. 2014; Pretorius et al. 2000). Ug99 is spreading through Africa and toward the Middle East and Asia, which have extensive wheat production, where it is causing significant crop losses (Singh et al. 2015). Stem rust $R$ genes that were initially effective against Ug99 have been overcome by new derivative Ug99 lineages (Jin et al. 2008, 2009; Singh et al. 2015). Other race-specific $R$ genes provide adequate resistance to Ug99 and its derivatives; however, it seems likely that, in time, many of these also will be overcome by one or more new races of Ug99. Although deploying partial resistance and nonrace-specific $R$ genes may help control Ug99 (Singh et al. 2015), new forms of resistance such as nonhost resistance that confer broad and durable resistance to wheat stem rust would be of great value for protecting global wheat production.

The model grass Brachypodium distachyon has been used for many studies of host-pathogen interactions (Fitzgerald et al. 2015). The species has been found to be a nonhost to the major cereal rust pathogens (Figueroa et al. 2015; Omidvar et al. 2018). A range of studies has documented the presence of natural variation for the degree of nonhost resistance to rust species in $B$. distachyon (Ayliffe et al. 2013; Figueroa et al. 2013; Garvin 2011; Omidvar et al. 2018). This has been exploited to explore the molecular genetic basis of variation in the degree of nonhost resistance to both stripe rust (Bettgenhaeuser et al. 2018; Gilbert et al. 2018) and wheat stem rust (Della Coletta et al. 2019). A more detailed understanding of the molecular basis of nonhost resistance to the rusts in $B$. distachyon would be desirable because of the practical agricultural implications should it be transferrable to crops.

Mutagenesis has proven to be foundational to unraveling many biological processes in plants, including disease resistance (Østergaard and Yanofsky 2004). In this study, we report on the genetic characterization of an induced mutant of $B$. distachyon that exhibits compromised nonhost resistance to wheat stem rust and, subsequently, employ a DNA sequencing-based genomics strategy to identify the gene in which the molecular lesion that underlies the loss of resistance is located. Our findings implicate the correct regulation of jasmonate signaling as a central aspect of nonhost resistance to wheat stem rust in $B$. distachyon.

\section{RESULTS}

Segregation for wheat stem rust resistance suggests a single mutated locus in $\gamma$ AL6.

The $B$. distachyon genotype $\mathrm{Bd} 21$ has been previously identified as resistant to wheat stem rust infection (Figueroa et al.
2013; Della Coletta et al. 2019), and exhibits few necrotic lesions upon inoculation (highly resistant, infection type fleck = ; ) (Fig. 1A). To investigate this nonhost resistance further, we subjected $\mathrm{Bd} 21$ to $\gamma$ irradiation and screened seedlings for compromised resistance to wheat stem rust. One mutant, $\gamma$ AL6, showed a contrasting phenotype to $\mathrm{Bd} 21$, with many small pustules developing over the surface of the leaf (susceptible, infection type 3-) (Fig. 1B), and a selfed progeny from this mutant ( $\gamma$ AL6.2) was subsequently crossed to Bd21 to develop a mapping population of 126 recombinant inbred lines (RILs). These RILs were inoculated with $P$. graminis f. sp. tritici and, using traditional phenotypic classifications for wheat stem rust (Stakman et al. 1962), 65 were rated resistant, 49 were susceptible, and 12 were considered to be segregating because different infection types were present among plants in the same inoculation or in different replicate inoculations. The disease scores of resistant RILs ranged from 0 ; to ;3- whereas those of susceptible RILs ranged from 3-; to 3. Infection types 2 and 4 were not observed. A $\chi^{2}$ analysis supported the hypothesis that the mutant phenotype is controlled by a single locus $\left(\chi^{2}=2.24, P>0.1\right)$. All 49 RILs deemed true breeding for susceptibility to $P$. grami$n i s$ f. sp. tritici were also susceptible to $P$. graminis f. sp. phleipretense that infects timothy grass, and 60 of 65 RILs deemed true breeding for resistance to $P$. graminis f. sp. tritici were also resistant for $P$. graminis f. sp. phlei-pretense, with the 5 other RILs scored as segregating for $P$. graminis $\mathrm{f}$. sp. phlei-pretense. None of the RILs exhibited resistance to one of the formae speciales and susceptibility to the other, suggesting that the same mutation confers susceptibility to both formae speciales of the pathogen.

The phenotypic reaction types were converted to numerical scores (0 to 9 scale) and a grand mean score for each RIL across the three $P$. graminis f. sp. tritici inoculations was calculated from replicate means to observe the distribution of the phenotypes as a quantitative trait (Fig. 2). A bimodal distribution was observed, with resistant RIL scores ranging from 0 to 2.33
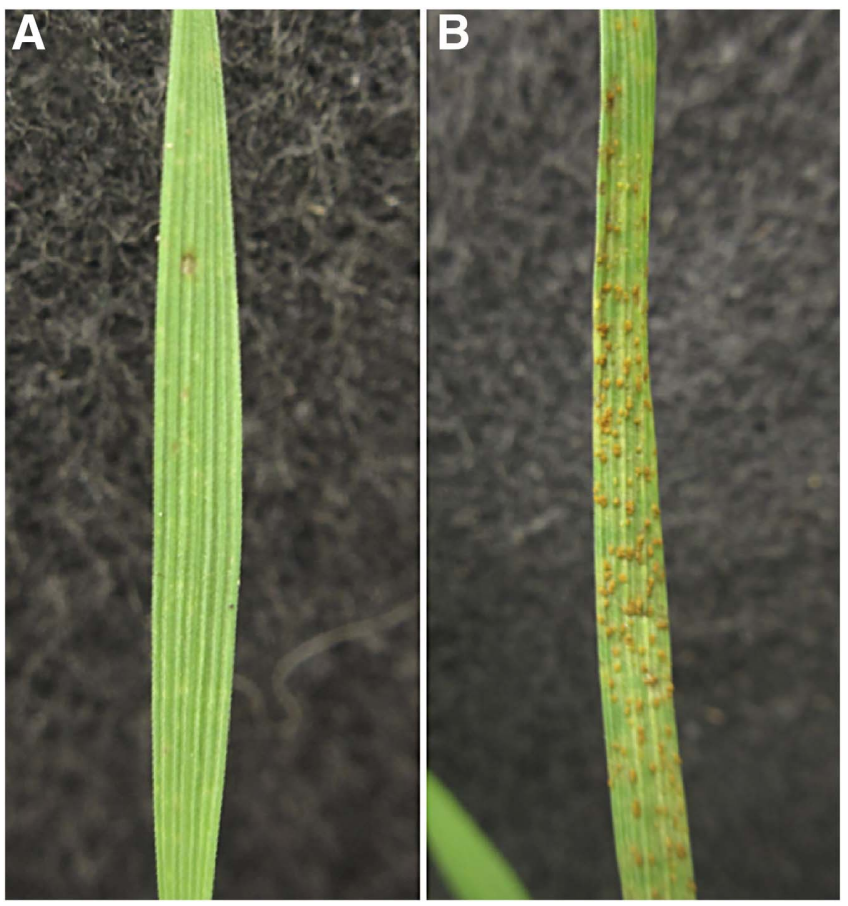

Fig. 1. Disease development on primary leaves of Brachypodium distachyon 9 days after inoculation with Puccinia graminis. A, Bd21 and B, selfed progeny of $\gamma$ AL6.2. 
and susceptible RIL scores from 3.79 to 7.67 . The average score of the $\mathrm{Bd} 21$ parent across three inoculations was 0.13 , ranging from 0 to 0.39 between replicates, whereas the average score of the $\gamma$ AL6.2 parent was 5.78, and ranged from 4.89 to 7 , depending upon the replicate. This further supported the involvement of a single major mutation underlying the difference in resistance in the population.

To examine the mutation's gene action, an $\mathrm{F}_{2}$ population from a cross between $\mathrm{Bd} 21$ and $\gamma$ AL6.3.1 (true-breeding $\mathrm{M}_{4}$ line derived from a full sib of $\gamma$ AL6.2) was evaluated. The results revealed subsets of individuals that fell near the values of the parents, presumably representing homozygous individuals. On the other hand, 117 of $187 \mathrm{~F}_{2}$ individuals fell between these homozygous peaks and were presumed to be heterozygotes. The two major classes of presumed heterozygotes were scored either as ;3-, ;3, 3-; or 3; (Supplementary Fig. S1). This suggests that the mutation is neither dominant nor recessive but, instead, appears to act in an additive fashion.

\section{A 1-bp deletion at high frequency}

\section{in the susceptible bulked segregant analysis pool.}

We performed whole-genome sequencing in two contrasting pools of most resistant and most susceptible RILs for bulked segregant analysis (BSA) (Michelmore et al. 1991) in an attempt to identify the mutation that compromises wheat stem rust resistance in YAL6. Paired-end reads from each pool were mapped to the $\mathrm{Bd} 21$ reference genome sequence (the wild-type genotype that was subjected to mutagenesis leading to the identification of $\gamma$ AL6) using two different aligners, Bowtie 2 and BWA. Despite the similar number of total mapped reads, the number of properly mapped reads (i.e., reads from a pair mapped in the correct orientation, with the appropriate insert size, and on the same chromosome) by BWA was higher than those from Bowtie2 (Supplementary Table S1). However, in both instances we obtained high genome coverage for both pools (approximately $30 \times$ for the resistant pool and $43 \times$ for the susceptible pool), which is critical for accurate variant calling.

Because the type of the mutation causing stem rust susceptibility in $\gamma$ AL6 and its selfed progeny was not known, different analytical approaches were completed in the search for the causal mutation. Analyses were performed for a range of possible

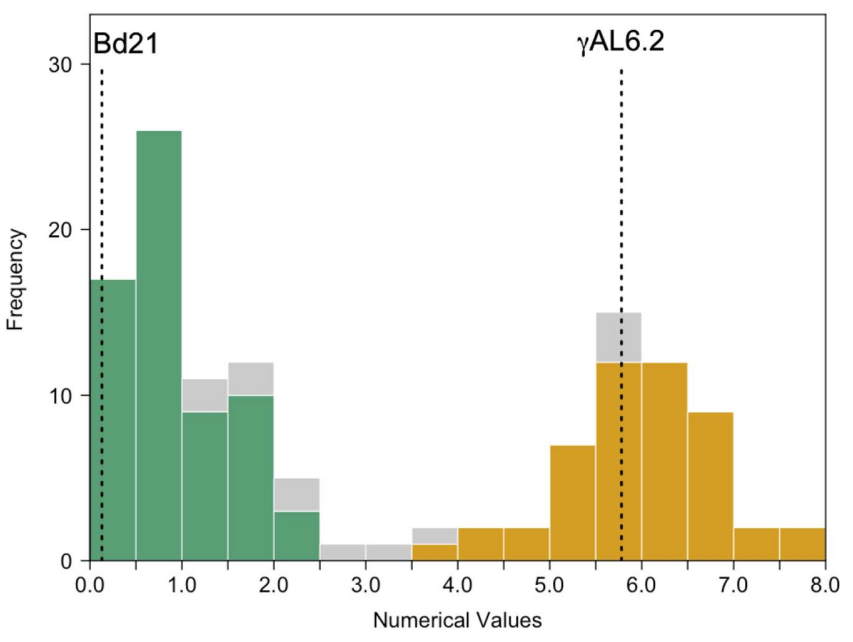

Fig. 2. Distribution of numerically converted phenotypes of $\mathrm{Bd} 21 \times$ $\gamma$ AL6.2 recombinant inbred line (RIL) population, averaged across three inoculations with Puccinia graminis. Green bars represent true-breeding resistant RILs, orange bars are true-breeding susceptible RILs, and gray bars are segregating RILs. Dashed lines represent the mean infection score for each parent, and the $\mathrm{x}$-axis ranges from the most resistant phenotype (infection type ;) to the most susceptible (infection type 3 ). outcomes, because the mutation could be a single-nucleotide polymorphism (SNP), a deletion, or other genome alterations.

The total number of high-quality SNPs identified between resistant and susceptible pools was only 1,704 in reads mapped with Bowtie2 and 1,027 with BWA. Their distribution across chromosomes and allele frequency differences between the BSA pools were examined for any genomic region in the susceptible pool with a high frequency of nonreference (susceptible) alleles. The cumulative SNP frequency plots did not identify any such regions (Supplementary Fig. S2). In agreement with this finding, we performed standard two-sided $Z$ tests and did not identify any genome region that had a significant difference in SNP allele frequency between resistant and susceptible pools.

The next approach taken was to examine structural variants that could underlie the stem rust susceptibility of $\gamma$ AL6. Because $\gamma$ irradiation in plants has historically been associated with the introduction of deletions (Sikora et al. 2011), this was the type of structural variant evaluated, with three different bioinformatics tools (Supplementary Table S2). For the BreakDancer tool, there were large differences in the number of deletions called, depending on the type of aligner used, mostly because this program called deletions in BWA mapped reads even when there were very few supporting reads. This was not an issue when Bowtie2 was the aligner, because all deletions called had more than 50 reads supporting it. Deletions detected in both resistant and susceptible BSA pools were discarded, and visual inspection of the remaining variants independently identified by BWA and Bowtie 2 with the Integrative Genomics Viewer (IGV) indicated that none of the deletions called in the susceptible pool appeared even remotely close to being homozygous (Supplementary Fig. S3).

Although program Delly did not identify any structural variants with reads aligned with Bowtie2, 109 deletions were called for the resistant pool and 199 for the susceptible pool when using BWA as the aligner, with 7 variants unique to the resistant pool and 95 unique to the susceptible pool. All were evaluated in IGV and found to be false positives.

Pindel was the last program used because it focuses on identification of small deletions. After mapping reads with Bowtie2, 174 deletions were called uniquely in the resistant pool and 609 uniquely in the susceptible pool. Deletions were discarded after visualization on IGV when homozygous or heterozygous deletions were present in both resistant and susceptible pools and when a unique deletion of the susceptible pool was heterozygous (Supplementary Fig. S4). IGV evaluation of all deletions revealed a 1-bp deletion on chromosome 1 that appeared nearly homozygous in the susceptible pool (Fig. 3). Of the 42 reads spanning this deletion, 38 had the deletion, 1 had an SNP at the position, and the other 3 were wild type. In contrast, in the resistant pool, 24 of 25 reads spanning this position did not have the deletion, while the other read did. When reads were mapped instead with BWA, this deletion was also found to be nearly fixed in the susceptible pool (37 of 42 reads), while the resistant RIL pool was homozygous for the wild-type allele ( 25 reads). Importantly, the closest variants to this deletion, an SNP $508 \mathrm{~kb}$ upstream and an SNP $367 \mathrm{~kb}$ downstream, had allele frequency differences between pools further from homozygosity $(0.33$ and 0.18 , respectively) (Supplementary File S1), indicating that the closest SNP variants in the region were in weaker linkage disequilibrium with the mutant phenotype than the deletion.

Subsequently, sequence data for each individual RIL in both BSA pools was obtained from PCR amplicons spanning the deletion. In the resistant pool, 41 of the RILs were homozygous for the wild-type allele, while the other 2 RILs were heterozygous. The detection of heterozygotes provides a likely explanation for why a read with the deletion was detected with Bowtie2 in the resistant BSA pool, and probably reflects a phenotyping error. In the susceptible pool, all 44 RILs were homozygous for 


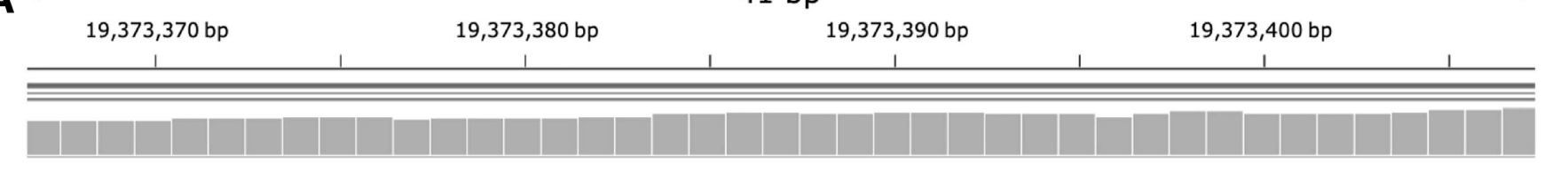

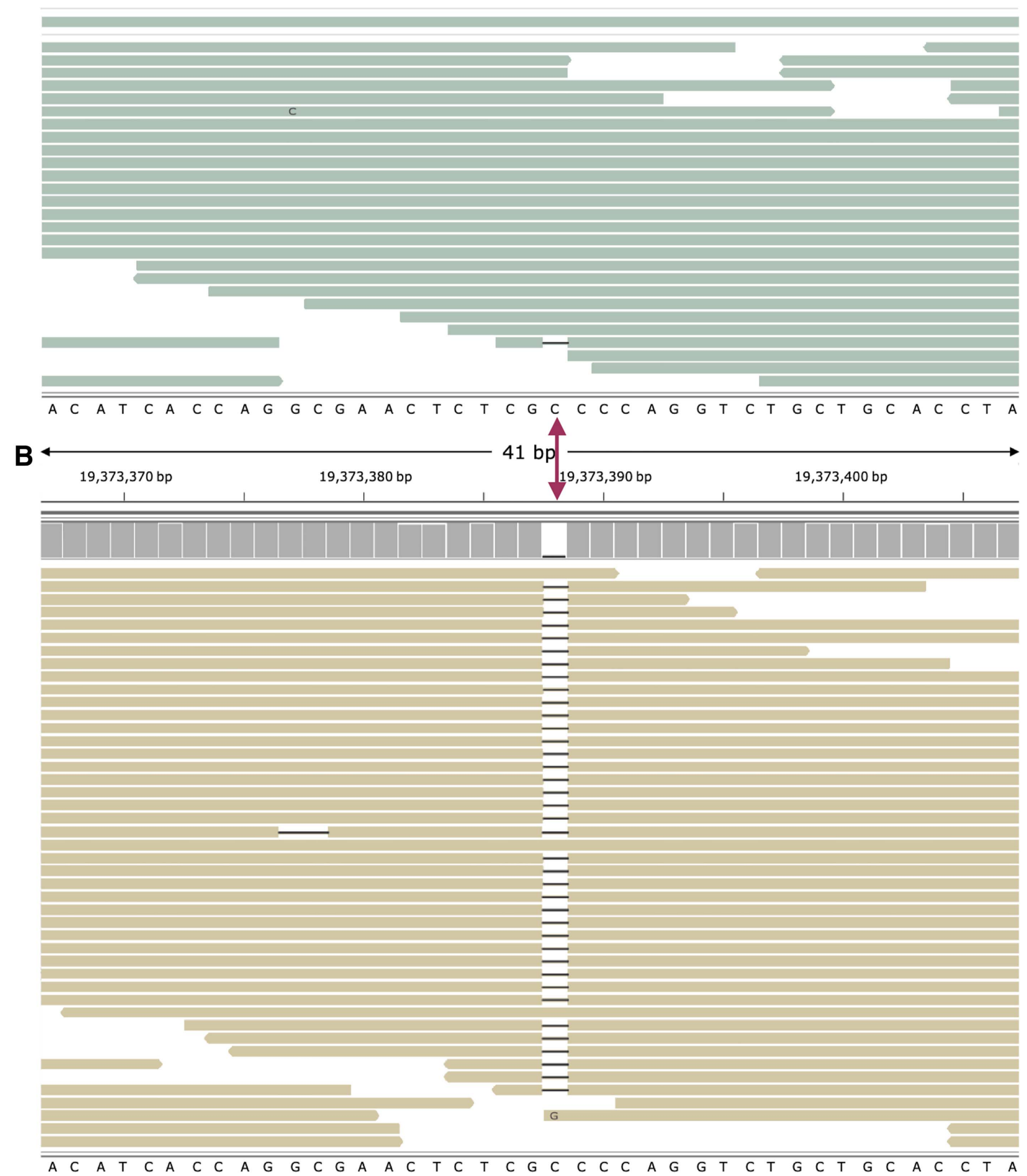

Fig. 3. Genomic region with a putative 1-bp deletion detected by Pindel on chromosome 1. A, Resistant pool and B, susceptible pool. Colored horizontal bars represent sequencing reads, black horizontal lines indicate deletion of bases, and gray vertical bars represent coverage at a single base. Red arrow indicates base position of interest. 
the deletion, suggesting that the wild-type reads detected originally in the susceptible BSA pool derived from these individuals might be bioinformatic artifacts, or possibly arose from tissue cross-contamination during harvests and pooling for BSA. Thus, this single base deletion appears to be the causal mutation conferring stem rust susceptibility to $\gamma$ AL6 and its progeny.

As additional validation, we performed whole-genome sequencing of $\gamma$ AL6.2, and the only homozygous variants detected within $\pm 1 \mathrm{Mb}$ of the single base deletion we detected were a 1-bp insertion $675 \mathrm{~kb}$ upstream and a separate 5-bp deletion $9.7 \mathrm{~kb}$ also upstream (average coverage in this region was approximately $34 \times$ ). These insertions-deletions (indels) are fixed in both the resistant and susceptible pools, indicating that neither cosegregates with susceptibility and, therefore, reflects falsepositive variants that likely were called due to small errors in the reference genome assembly (Supplementary Fig. S5). This also explains why they were not detected in the original BSA analysis. However, given the proximity to our putative causal mutation, we also performed Sanger sequencing on PCR fragments spanning the 5-bp deletion and confirmed that it is present and homozygous in Bd21, $\gamma$ AL6.2, and both resistant and susceptible pools. Therefore, we have strong evidence that the single base pair deletion on chromosome 1 , and not a different variant, is the causal mutation causing susceptibility to wheat stem rust.

\section{yAL6.2 contains a 1-bp deletion in Bradi1g24100, a} homolog of the Arabidopsis TIME FOR COFFEE gene.

The deletion identified by BSA is located in the coding sequence (CDS) of the gene Bradilg24100. In B. distachyon genome annotation v3.1 (Department of Energy Joint Genome Institute), this gene model is functionally annotated as a nuclear hormone receptor binding (Blast2GO). There are no paralogs in other regions of the $B$. distachyon genome. Comparison of this gene to homologs of other species revealed that the gene with the highest homology in the Arabidopsis genome is AT3G22380 (Supplementary Fig. S6), designated TIME FOR COFFEE (TIC) (Hall et al. 2003). The EMBOSS Needle Pairwise Sequence Alignment shows that the protein sequences from these two species share $42.3 \%$ similarity, with $29.8 \%$ of the amino acid residues being identical. Sequencing of $\gamma$ AL6.2 progeny indicated that it, too, has the deletion detected in the susceptible BSA pool, at position 19,373,388 bp on chromosome 1, whereas $\mathrm{Bd} 21$ does not (Fig. 4). Translation of the Bd21 CDS with the ExPASy online tool revealed that the wildtype protein sequence contains 1,634 residues, while the single base deletion in $\gamma$ AL6.2 causes a frame shift at amino acid residue 1,282 that introduces a premature stop codon at residue 1,296 (Supplementary Fig. S7).

\section{DISCUSSION}

In this study, nonhost resistance to stem rust was explored using mutagenesis, genetics, and genomics coupled with bioinformatic analysis in the model grass $B$. distachyon. This species has been found to be of value for exploring numerous interactions between plants and pathogens (Fitzgerald et al. 2015). This includes the major grass rusts (Ayliffe et al. 2013; Draper et al. 2001; Figueroa et al. 2013; Omidvar et al. 2018) as well as the adapted species $P$. brachypodii (Barbieri et al. 2011). The close evolutionary relationship between $B$. distachyon, itself a coolseason grass, and the cool-season cereals makes it particularly amenable for studies of nonhost resistance to these rusts. In contrast to $B$. distachyon, studies of the interaction between rice and rust pathogens revealed that the degree of nonhost resistance is very high and differences between genotypes are not visible to the eye (Ayliffe et al. 2011).
To complement information recently gained from molecular genetic analyses of natural variation in nonhost resistance to rust pathogens (Bettgenhaeuser et al. 2018; Della Coletta et al. 2019; Gilbert et al. 2018), induced mutagenesis was employed in this study to generate unique mutations in genes that are critical for nonhost resistance to stem rust. Mutagenesis has been broadly used for over a century in the plant kingdom for practical purposes such as crop breeding (Maluszynski and Szarejko 2005; Rutger 1992) as well as more basic uses such as for unraveling the molecular basis of biological processes (Østergaard and Yanofsky 2004). $\gamma$ AL6 was initially identified based on its susceptibility to $P$. graminis $\mathrm{f}$. $\mathrm{sp}$. phlei pretense, to which $\mathrm{Bd} 21$ is resistant (Figueroa et al. 2013; Garvin 2011), and to P. graminis f. sp. tritici, which causes wheat stem rust. Mutagenesis previously has been used to identify $B$. distachyon mutants with altered resistance to wheat stripe rust, to which $B$. distachyon appears to be a near nonhost (An et al. 2016). However, in that study, the mutagenizing agent was insertional mutagenesis facilitated by Agrobacterium tumefaciens, which has been used to generate large numbers of mutants in B. distachyon (Bragg et al. 2012).

Genetic analysis of wheat stem rust resistance in a RIL population from a cross between $\gamma$ AL6 and Bd21 suggested that the causative mutation underlying the stem rust susceptibility of $\gamma$ AL6 was associated with a single locus. The high correlation between phenotypic rust resistance and susceptibility scores of the RILs after infection with $P$. graminis f. sp. tritici and $P$. graminis f. sp. phlei-pretense, and the fact that $\gamma$ AL6 also appears to be far more susceptible to oat stem rust (Supplementary Fig. S8),
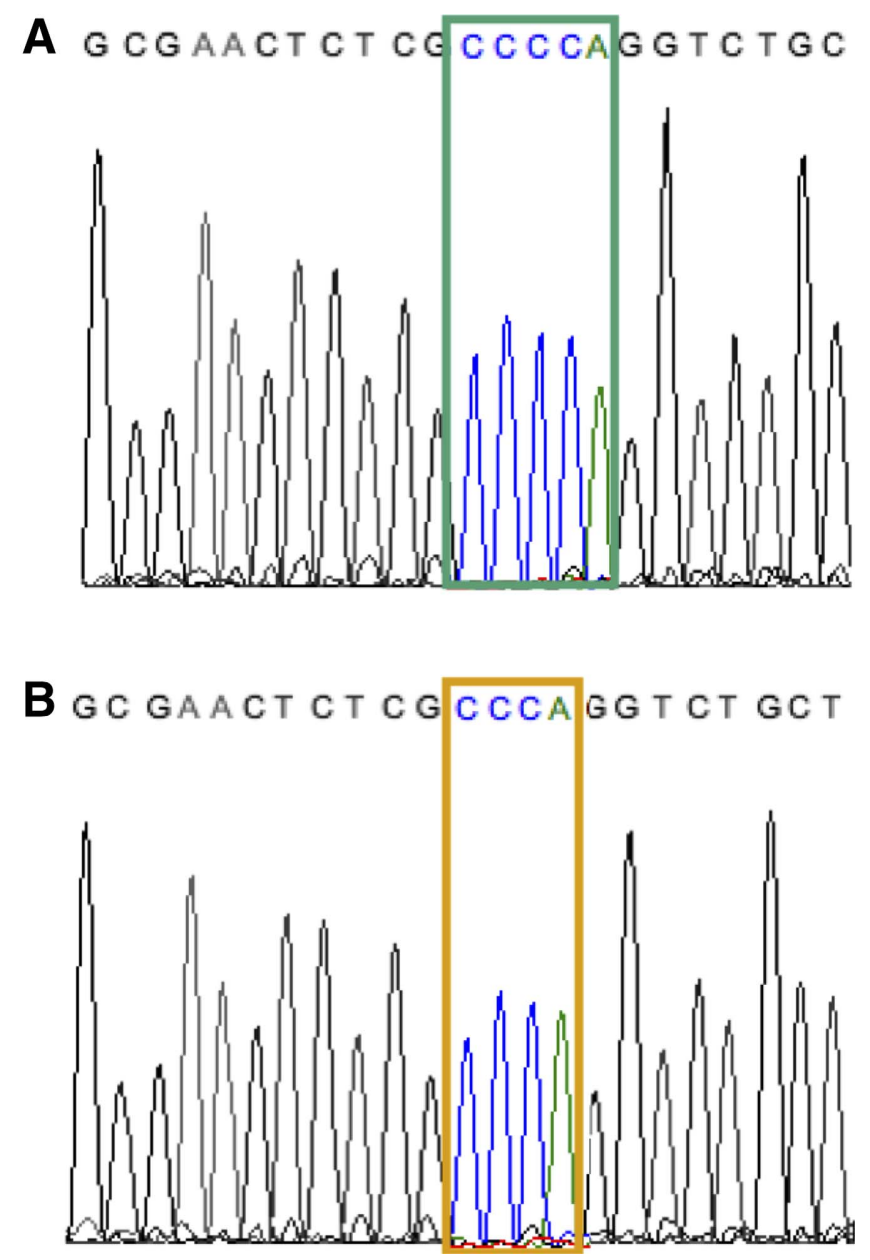

Fig. 4. DNA sequence chromatogram comparison between $\mathbf{A}, \mathrm{Bd} 21$ and B, $\gamma$ AL6.2, displaying a single-base deletion in $\gamma$ AL6.2. 
suggested that the mutation involved a gene broadly critical to nonhost resistance to $P$. graminis. Evaluation of $\mathrm{F}_{2}$ populations to determine the mode of action of the mutation suggests that a single copy of the wild-type allele is not sufficient to maintain the nonhost resistance of $\mathrm{Bd} 21$.

BSA employing two pools containing RILs with susceptible versus wild-type (resistant) phenotypes was employed to identify a potential causal mutation in $\gamma$ AL6 that results in the loss of nonhost resistance. This 30-year-old method (Michelmore et al. 1991) has proven remarkably adaptable to advances in genomic technologies as an efficient strategy to map genes and mutations. With the drop in nucleic acid sequencing cost, coupled with next-generation sequencing technology and high-quality reference genome sequences and bioinformatics tools, BSA has been effectively used to identify the location of mutations that cause phenotypic changes (Abe et al. 2012; Austin et al. 2011; Dobbels et al. 2017, Schneeberger et al. 2009).

In this study, the $B$. distachyon reference genome sequence is that of the wild-type genotype $\mathrm{Bd} 21$, which simplified bioinformatic analysis. Few high-quality SNPs were identified and no large differences in SNP allele frequency between the pools were identified, suggesting that the causal mutation was not an SNP. Radiation often induces deletions (Sikora et al. 2011) that can be challenging to identify with short-read next-generation sequencing. Thus, several different programs were employed to search for deletions in the BSA pools. All deletions detected by BreakDancer and Delly were found to be false positives. Pindel also identified many false positives but a 1-bp deletion was detected at very high frequency in the susceptible BSA pool that was nearly absent in the resistant pool. Sanger sequencing of each individual making up the resistant and susceptible pools revealed that all but two of the resistant RILs were homozygous for the wild-type allele, with two being heterozygous, while all susceptible RILs were homozygous for the deletion. Progeny of $\gamma$ AL6 also harbor this deletion, whereas $\mathrm{Bd} 21$ does not. Although $\gamma$ irradiation is often thought to introduce large genome mutations, other researchers also observed small deletions, as small as $1 \mathrm{bp}$ in $\gamma$ irradiation-induced mutants of Arabidopsis and rice (Cecchini et al. 1998; Finkelstein et al. 1998; Sato et al. 2009). In this study, the identification of such a small deletion highlights the power of next-generation sequencing to detect mutations; the facts that the $B$. distachyon genome is so compact, the reference genome sequence is very high quality, and the reference genome genotype was the genotype selected for mutagenesis facilitated our analysis.

The single-base deletion in $\gamma$ AL6 introduces a premature stop codon in the protein sequence of the gene Bradilg24100, annotated as a nuclear hormone receptor-binding protein and a homo$\log$ of the Arabidopsis gene At3g22380, known as TIC. This gene, first identified in an EMS mutant (Hall et al. 2003) and later isolated and found to act in the nucleus (Ding et al. 2007), was found to be an important regulator of the circadian clock. Subsequently it has been found to play a central role in elements regulating numerous other biological processes associated with development, metabolism, and environmental adaptation (Sanchez-Villarreal et al. 2013; Shin et al. 2013).

Clues to the biological basis of susceptibility attributable to the mutation in BdTIC may be found in results of a prior study by Shin et al. (2012). They reported that, in Arabidopsis, TIC regulates jasmonate (JA) signaling by negatively regulating the accumulation of the protein MYC2, a master positive transcriptional regulator of JA responses in the JA pathway (Robert-Seilaniantz et al. 2011), through MYC2 protein degradation. Without proper regulation of MYC2 protein levels, an excess of this central positive regulator of the JA pathway accumulates. In Arabidopsis tic mutants, proliferation of the hemibiotrophic bacterium $P$. syringae increased 30-fold compared with the wild type after infection (Shin et al. 2012) and was attributed to a loss of TIC-mediated negative regulation of JA signaling. $P$. syringae exploits the antagonism between the JA pathway and the salicylic acid (SA) pathway to infect a host by producing the JA mimic coronatine, which activates the JA pathway and leads to the suppression of the SA pathway (Zhao et al. 2003) that would normally confer resistance. The observations of Shin et al. (2012) suggest that the loss of TIC protein-mediated degradation of MYC2 leads to elevated JA pathway-based suppression of the SA pathway beyond the action of coronatine alone. Therefore, we speculate that the mutation in the TIC homolog that we have identified and now term BdTIC may result in a similar outcome; namely, that this mutation alters JA pathway homeostasis sufficiently to disrupt nonhost resistance to $P$. graminis. However, this is just one possible explanation of how the mutation may be disrupting nonhost resistance because TIC regulates so many cellular processes. Further research will shed light on this issue.

Recent research on the interaction between $B$. distachyon and $P$. coronata, the causal pathogen of oat crown rust, supported a potential role for the SA pathway as well as ethylene signaling, but not the JA pathway, in nonhost resistance to that pathogen, based on expression analysis of different well-known defenserelated genes (Omidvar et al. 2018). Our results comport with this previous research. As such, our results suggest a possible role for SA-mediated ETI as one component of the nonhost resistance of $B$. distachyon to stem rust, which requires finely tuned crosstalk between the SA pathway and the JA pathway. This proposed involvement of hormone homeostasis in nonhost resistance comports with the recent proposal that nonhost resistance does not necessarily need to involve unique immune processes but, rather, relies on existing components of plant immunity (Panstruga and Moscou 2020). Therefore, the rAL6 mutant may be a useful resource for further exploration of various molecular aspects of nonhost resistance to stem rust in this species, as well as the phenomenon of nonhost resistance in general.

In conclusion, this study identified a 1-bp deletion within the gene Bradilg24100, a homolog of the Arabidopsis gene TIC, in an induced mutant of $B$. distachyon. The mutation disrupts nonhost resistance to three formae speciales of Puccinia. Based on the prior observation that disrupting TIC in Arabidopsis greatly enhances susceptibility to a bacterial hemibiotroph, our result provides evidence that disrupting its ortholog in $B$. distachyon leads to the loss of nonhost resistance to an obligate fungal biotroph. This finding may suggest that proper modulation of the JA and SA pathways at the time of infection by $P$. graminis is critical for nonhost resistance to stem rust in B. distachyon.

\section{MATERIALS AND METHODS}

Plant materials and stem rust inoculations.

Seed of $B$. distachyon inbred line $\mathrm{Bd} 21$ were subjected to 200 gray of $\gamma$ irradiation. One hundred $\mathrm{M}_{1}$ seeds were then planted and selfed to obtain $\mathrm{M}_{2}$ seeds. Approximately $1,150 \mathrm{M}_{2}$ seeds were sown (16 seeds per $6.3-\mathrm{cm}$ pot) and placed in a growth chamber at $25^{\circ} \mathrm{C}$ and $14 \mathrm{~h}$ of light. After 7 days, seedlings were inoculated with spores of $P$. graminis $\mathrm{f}$. sp. phlei-pretense (timothy stem rust pathogen) obtained from the United States Department of Agriculture-Agricultural Research Service Cereal Disease Laboratory and increased on the timothy cultivar Toro using the general inoculation method described by Della Coletta et al. (2019). This $P$. graminis f. sp. phlei-pratensis was used because it is capable of infecting certain susceptible genotypes of B. distachyon (Figueroa et al. 2013). Plants were monitored for evidence of altered stem rust resistance between 7 and 9 days after inoculation. One of the $\mathrm{M}_{2}$ plants, designated $\gamma$ AL6, exhibited compromised resistance. Although $\mathrm{Bd} 21$ is resistant to this pathogen, sporulating pustules developed on $\gamma$ AL6. An $\mathrm{M}_{3}$ 
progeny test of $\gamma$ AL6 suggested that it was true breeding for the susceptible phenotype. Subsequently, an $\mathrm{F}_{5: 6}$ population of RILs from a cross between a single $\mathrm{M}_{3}$ plant retained from the progeny test ( $\gamma$ AL6.2) and $\mathrm{Bd} 21$ was developed, as was an $\mathrm{F}_{2}$ population from crossing $\mathrm{Bd} 21$ and $\gamma \mathrm{AL6}$ 6.3.1, the latter being an $\mathrm{M}_{4}$ plant derived from selfing a second $\mathrm{M}_{3}$ full sib of $\gamma$ AL6.2 $(\gamma \mathrm{AL6.3)}$ that was also retained from the progeny test.

\section{Wheat stem rust inoculation and disease evaluation.}

$P$. graminis f. sp. tritici urediniospores were obtained from 4week-old wheat cultivar McNair and used to inoculate the RIL population, as detailed by Della Coletta et al. (2019). Briefly, four RILs were planted in quadrants within $6.3-\mathrm{cm}$ square pots ( 5 seeds/RIL), which were then placed in a tray ( 6 pots/tray). In each tray, 5 seeds from $\mathrm{Bd} 21$ and $\gamma \mathrm{AL} 6.2$ were also planted throughout to serve as controls. After 5 days of stratification at $5^{\circ} \mathrm{C}$, the trays were transferred to a growth chamber set to run in a growth chamber at $24^{\circ} \mathrm{C}$ with a 14-h day length for 7 days until inoculation.

For inoculations, spores $(5 \mathrm{mg})$ of $P$. graminis f. $\mathrm{sp}$. tritici genotype 78-21-BB463 ( $P$. graminis f. sp. tritici 4a) (Zambino et al. 2000) race DFBJ (Zhong et al. 2009), harvested between 1 and 6 days prior to inoculation, were suspended in $500 \mu \mathrm{l}$ of Soltrol 170 oil and pneumatically sprayed onto each tray of $B$. distachyon seedlings. Plants were then transferred to a growth chamber set at $20^{\circ} \mathrm{C}$ for overnight misting in the dark for 17.5 $\mathrm{h}$ and, following $2 \mathrm{~h}$ of light the next morning at $22^{\circ} \mathrm{C}$, were moved back to the growth chamber. Disease development was evaluated 9 days later. The wheat stem rust scoring method developed by Stakman et al. (1962) was employed to score the infection type of $B$. distachyon seedlings. Disease evaluations were replicated three times per RIL with $P$. graminis f. sp. tritici 4a and once with $P$. graminis f. sp. phlei-pretense. An average phenotypic infection type for each RIL was obtained, and infection types were converted to numerical values between 0 (most resistant) and 9 (most susceptible) (Gao et al. 2016).

Additionally, an $\mathrm{F}_{2}$ population from the cross between $\gamma$ AL6.3.1 and $\mathrm{Bd} 21$ was evaluated for wheat stem rust resistance to examine the mutation's gene action. Inoculations were the same as described for the RILs, with the following modifications: nine $F_{2}$ seeds were planted in each pot, with three seeds of each parent randomly planted per tray. In total, $187 \mathrm{~F}_{2}$ seedlings was evaluated over three inoculations.

\section{BSA sequencing.}

After the second disease evaluation, infected leaves were removed and pathogen-free leaves were harvested 10 days later for DNA extraction and sequencing. We selected 43 RILs for the resistant pool and 44 RILs for the susceptible pool based on their average phenotypic infection type after inoculation with $P$. graminis f. sp. tritici 4a and harvested one leaf of approximately equal size per RIL. The leaves for individual RILs representing each pool were combined and DNA was extracted from each BSA pool (Qiagen DNeasy Plant Mini), and submitted for sequencing library preparation with the TruSeq nano kit by the University of Minnesota Genomics Center (UMGC) (DNA input of $357.5 \eta \mathrm{g}$ and $382.5 \eta \mathrm{g}$ for resistant and susceptible pools, respectively). Finally, 100-bp paired-end reads were generated by the Illumina HiSeq 2500 sequencer.

\section{Bioinformatic analysis.}

We performed a series of quality control procedures with the raw sequencing reads and used FastQC v0.11.2 to visualize the results at each step. First, Illumina sequencing adapters from both ends were removed with CutAdapt v.1.8.1 (Martin 2011) and only reads longer than 40 bp were kept (option '-m 40'). Second, low-complexity reads were removed using 'fastx_artifacts_filter' from FASTX toolkit v.0.0.14. The first $9 \mathrm{bp}$ of most reads of the susceptible pool were very low quality with a high k-mer content; thus, they were removed using 'fastx_trimmer' from the FASTX toolkit (option '-f 10'). Also, low-quality bases from the ends of reads from both pools were removed with 'fastq_quality_trimmer', keeping only reads longer than $40 \mathrm{bp}$. Finally, high-quality pairedend reads were synced using a custom python script developed by M. Vermaat and freely available at GitHub.

Filtered and synced reads from both pools were mapped to the reference genome sequence of $B$. distachyon (v3.1 available at Phytozome 12) using Bowtie2 v2.2.4 (Langmead and Salzberg 2012) and also with BWA v0.7.10 (Li 2013). It should be noted that this reference genome sequence is that of the wild-type parent, $\mathrm{Bd} 21$. Because there was no way to know what type of mutation caused the loss of resistance in $\gamma$ AL6.2, two different aligners were employed to reduce possible mapping bias. Paired-end reads were mapped using 'end-to-end' and 'very-sensitive' modes of Bowtie2, and also using the algorithm 'mem' from BWA. The number of mapped reads were calculated using the program 'stats' from BAMTools v.2.1.1, and potential duplicated reads were removed using the program 'MarkDuplicates' from Picard v1.133.

Comparative analysis of the BSA RIL pools to identify genome variants present in the susceptible pool but absent in the resistant pool began with an examination of SNPs. SAMTools v1.2 'mpileup' and BCFTools v1.2 'bcftools call' with 'consensus-caller' method were used to call SNPs with both pools at the same time in order to get the allele frequencies for the same position. SNPs with low mapping quality (option '-Q 30 ') and with read depth between 10 and 70 reads were filtered out with 'vcfutils.pl varFilter' (SAMTools v1.2). The program 'bcftools filter' removed SNPs that were within 5 bp of an indel (option '-g 5'), SNPs with fewer than 5 reads supporting the reference and alternate allele, and SNPs with genotype quality smaller than 30. Allele frequencies were calculated with a custom python script and then visualized in a plot with a 5-SNP window size (rolling every $1 \mathrm{SNP}$ ) generated by a modified version of the R script from the Genomics, Evolution and Development Lab at Michigan State University. Finally, we modified a custom R script developed by Haase et al. (2015) to perform standard two-sided $Z$ tests averaged in 5-SNP windows and determine whether any genomic region had statistically significant (false discovery rate adjusted $P$ values $<0.05$ ) allele frequency different between resistant and susceptible pools.

Structural variants were also evaluated, which is complicated by the short read lengths from next-generation sequencing. There are many programs available for this purpose and using more than one such software program is recommended to decrease the number of false positives (Kosugi et al. 2019; Pabinger et al. 2014). Thus, three programs were used to identify structural variants: BreakDancer v1.1 (cpp version) (Chen et al. 2009), Delly v0.7.2 (Rausch et al. 2012), and Pindel v0.2.5b6 (Ye et al. 2009).

With BreakDancer, a configuration file for both BSA RIL pools was generated with information about insert sizes of paired-end reads with high mapping quality (option '-q 30') and cut off in 3 standard deviation (option '-c 3'). The configuration files generated were then used to call variants with the program 'breakdancer_max' with minimum mapping quality of 30 (option '-q 30'), minimum output score of 90 (option '-y 90') and maximum coverage of 200 reads (option '-x 200'). For Delly, variants were called from reads that had with high mapping quality (option '-q 30'). For Pindel, only variants with minimum of 5 and maximum of 100 supporting reads were reported (options '-e 5 -f 100'). Finally, for each program structural variants detected in both BSA RIL pools were discarded. The remaining variants were visualized with the IGV (Robinson et al. 2011) to confirm variants present in the susceptible but not resistant pool and to discard false positives. 


\section{Confirming a putative causal mutation in $\gamma$ AL6.2.}

Sanger sequencing was used to verify that a mutation detected at high frequency in the susceptible but not the resistant BSA pool was present in progeny of $\gamma$ AL6.2 but not Bd21. A DNA sequence flanking $250 \mathrm{bp}$ of the mutation was extracted from the reference genome sequence with a custom python script, and primers were designed with Primer3 to yield a PCR product size of $300 \mathrm{bp}$ with the mutation near the middle of the product, then validated with reverse electronic PCR v.2.3.12 (Rotmistrovsky et al. 2004; Schuler 1997).

The genomic region containing the mutation was amplified by PCR prior to sequencing. PCR assays ( $20 \mu$ volume) were set up for Bd21 and $\gamma$ AL6.2 DNA (extracted as described above), containing $2 \mu \mathrm{l}$ of $10 \times$ PCR Buffer (Applied Biosystems), $0.4 \mu \mathrm{l}$ of $10 \mathrm{mM}$ dNTPs, $2 \mu \mathrm{l}$ of forward and reverse primers $(1 \mu \mathrm{M}$ stock concentration), $0.1 \mu \mathrm{l}$ of Taq DNA Polymerase $(5 \mathrm{U} / \mu \mathrm{l})$, and 10 ng of DNA. PCR conditions consisted of initial denaturation at $93^{\circ} \mathrm{C}$ for $3 \mathrm{~min} ; 35$ cycles of denaturation at $93^{\circ} \mathrm{C}$ for $30 \mathrm{~s}$, annealing at $50^{\circ} \mathrm{C}$ for $30 \mathrm{~s}$, and extension at $72^{\circ} \mathrm{C}$ for $50 \mathrm{~s}$; and final extension at $72^{\circ} \mathrm{C}$ for $5 \mathrm{~min}$. The PCR products were evaluated on a $1 \%$ agarose gel to confirm amplification, then purified using the QIAQuick PCR Purification Kit according to the manufacturer's instructions. After Nanodrop DNA quantification, parental PCR amplicons were sent to UMGC for Sanger sequencing. Subsequently, DNA from each individual RIL included in both BSA pools was used for PCR using the same PCR amplifications as described above and sent to Genewiz for PCR purification and Sanger sequencing with the forward primer.

Finally, we sequenced the genome of $\gamma$ AL6.2 with Illumina MiSeq v3 as a final independent effort to identify variants in the mutant around the putative causal mutation. The 250-bp pairedend reads were subjected to the same quality control, mapping, and variant calling procedures as described above for BSA.

\section{ACKNOWLEDGMENTS}

We thank P. Gustafson for arranging seed $\gamma$ irradiation, Z. Blankenheim for technical assistance on this project, C. Hirsch for helpful discussions on the bioinformatic analyses completed in this study, and the Minnesota Supercomputing Institute (MSI) at the University of Minnesota for providing resources employed in this study.

\section{AUTHOR-RECOMMENDED INTERNET RESOURCES}

Babraham Bioinfomatics FastQC, v0.11.2:

https://www.bioinformatics.babraham.ac.uk/projects/fastqc

Broad Institute Picard v1.133: https://broadinstitute.github.io/picard

EMBOSS Needle Pairwise Sequence Alignment:

https://www.ebi.ac.uk/Tools/psa/emboss_needle

ExPASy online translation tool: https://web.expasy.org/translate

Genewiz website: https://www.genewiz.com

GitHub BAMTools v.2.1.1: https://github.com/pezmaster31/bamtools

GitHub BCFTools v1.2: https://github.com/samtools/bcftools

GitHub rust conversion script:

https://github.com/umngao/rust_scores_conversion

GitHub SAMTools v1.2: https://github.com/samtools/samtools

Hannon Lab FASTX toolkit v.0.0.14:

http://hannonlab.cshl.edu/fastx_toolkit/index.html

Phytozome 12 Brachypodium distachyon v3.1:

https://phytozome-next.jgi.doe.gov/

Primer3 tool: https://bioinfo.ut.ee/primer3-0.4.0

Python script by M. Vermaat for syncing paired-end reads: https://github. $\mathrm{com} / \mathrm{martijnvermaat/bio-playground/tree/master/sync-paired-end-reads}$

SNP frequency graphing: https://github.com/ngs-docs/edda/blob/master/ doc/tutorials-2012/files/plot_allele_freq_data.R

\section{LITERATURE CITED}

Abe, A., Kosugi, S., Yoshida, K., Natsume, S., Takagi, H., Kanzaki, H., Matsumura, H., Yoshida, K., Mitsuoka, C., Tamiru, M., Innan, H., Cano, L., Kamoun, S., and Terauchi, R. 2012. Genome sequencing reveals agronomically important loci in rice using MutMap. Nat. Biotechnol. 30:174-178.

An, T., Cai, Y., Zhao, S., Zhou, J., Song, B., Bux, H., and Qi, X. 2016 Brachypodium distachyon T-DNA insertion lines: A model pathosystem to study nonhost resistance to wheat stripe rust. Sci. Rep. 6: 25510.

Austin, R. S., Vidaurre, D., Stamatiou, G., Breit, R., Provart, N. J., Bonetta, D., Zhang, J., Fung, P., Gong, Y., Wang, P. W., McCourt, P., and Guttman, D. S. 2011. Next-generation mapping of Arabidopsis genes. Plant J. 67:715-725.

Ayliffe, M., Devilla, R., Mago, R., White, R., Talbot, M., Pryor, A., and Leung, H. 2011. Nonhost resistance of rice to rust pathogens. Mol. Plant-Microbe Interact. 24:1143-1155.

Ayliffe, M., Singh, D., Park, R., Moscou, M., and Pryor, T. 2013. Infection of Brachypodium distachyon with selected grass rust pathogens. Mol. Plant-Microbe Interact. 26:946-957.

Barbieri, M., Marcel, T. C., and Niks, R. E. 2011. Host status of false brome grass to the leaf rust fungus Puccinia brachypodii and the stripe rust fungus $P$. striiformis. Plant Dis. 95:1339-1345.

Bettgenhaeuser, J., Gardiner, M., Spanner, R., Green, P., Hernández-Pinzón, I., Hubbard, A., Ayliffe, M., and Moscou, M. J. 2018. The genetic architecture of colonization resistance in Brachypodium distachyon to non-adapted stripe rust (Puccinia striiformis) isolates. PLoS Genet. 14: e1007637.

Bettgenhaeuser, J., Gilbert, B., Ayliffe, M., and Moscou, M. J. 2014. Nonhost resistance to rust pathogens-A continuation of continua. Front. Plant Sci. 5:664

Boller, T., and Felix, G. 2009. A renaissance of elicitors: Perception of microbe-associated molecular patterns and danger signals by patternrecognition receptors. Annu. Rev. Plant Biol. 60:379-406.

Bragg, J. N., Wu, J., Gordon, S. P., Guttman, M. E., Thilmony, R., Lazo, G. R., Gu, Y. Q., and Vogel, J. P. 2012. Generation and characterization of the Western Regional Research Center Brachypodium T-DNA insertional mutant collection. PLoS One 7:e41916.

Cecchini, E., Mulligan, B. J., Covey, S. N., and Milner, J. J. 1998. Characterization of gamma irradiation-induced deletion mutations at a selectable locus in Arabidopsis. Mutat. Res. 401:199-206.

Chen, K., Wallis, J. W., McLellan, M. D., Larson, D. E., Kalicki, J. M., Pohl, C. S., McGrath, S. D., Wendl, M. C., Zhang, Q., Locke, D. P., Shi, X., Fulton, R. S., Ley, T. J., Wilson, R. K., Ding, L., and Mardis, E. R. 2009. BreakDancer: An algorithm for high-resolution mapping of genomic structural variation. Nat. Methods 6:677-681.

Della Coletta, R., Hirsch, C. N., Rouse, M. N., Lorenz, A., and Garvin, D. F. 2019. Genomic dissection of nonhost resistance to wheat stem rust in Brachypodium distachyon. Mol. Plant-Microbe Interact. 32: 392-400.

Ding, Z., Millar, A. J., Davis, A. M., and Davis, S. J. 2007. TIME FOR COFFEE encodes a nuclear regulator in the Arabidopsis thaliana circadian clock. Plant Cell 19:1522-1536.

Dobbels, A. A., Michno, J. M., Campbell, B. W., Virdi, K. S., Stec, A. O., Muehlbauer, G. J., Naeve, S. L., and Stupar, R. M. 2017. An induced chromosomal translocation in soybean disrupts a KASI ortho$\log$ and is associated with a high-sucrose and low-oil seed phenotype. G3: Genes Genomes Genet. 7:1215-1223.

Dodds, P. N., and Rathjen, J. P. 2010. Plant immunity: Towards an integrated view of plant-pathogen interactions. Nat. Rev. Genet. 11:539548.

Draper, J., Mur, L. A. J., Jenkins, G., Ghosh-Biswas, G. C., Bablak, P., Hasterok, R., and Routledge, A. P. M. 2001. Brachypodium distachyon. A new model system for functional genomics in grasses. Plant Physiol. 127:1539-1555.

Ellis, J. G., Lagudah, E. S., Spielmeyer, W., and Dodds, P. N. 2014. The past, present and future of breeding rust resistant wheat. Front. Plant Sci. 5:641.

Figueroa, M., Alderman, S., Garvin, D. F., and Pfender, W. F. 2013. Infection of Brachypodium distachyon by formae speciales of Puccinia graminis: Early infection events and host-pathogen incompatibility. PLoS One 8:e56857.

Figueroa, M., Castell-Miller, C. V., Li, F., Hulbert, S. H., and Bradeen, J. M. 2015. Pushing the boundaries of resistance: Insights from Brachypodium-rust interactions. Front. Plant Sci. 6:558.

Finkelstein, R. R., Wang, M. L., Lynch, T. J., Rao, S., and Goodman, H. M. 1998. The Arabidopsis abscisic acid response locus ABI4 encodes an APETALA 2 domain protein. Plant Cell 10:1043-1054.

Fitzgerald, T. L., Powell, J. J., Schneebeli, K., Hsia, M. M., Gardiner, D. M., Bragg, J. N., McIntyre, C. L., Manners, J. M., Ayliffe, M., Watt, M., Vogel, J. P., Henry, R. J., and Kazan, K. 2015. 
Brachypodium as an emerging model for cereal-pathogen interactions. Ann. Bot. 115:717-731.

Gao, L., Turner, M. K., Chao, S., Kolmer, J., and Anderson, J. A. 2016. Genome wide association study of seedling and adult plant leaf rust resistance in elite spring wheat breeding lines. PLoS One 11: e0148671.

Garvin, D. F. 2011. Investigating rust resistance with the model grass Brachypodium. Pages 89-91 in: Proc. Borlaug Global Rust Initiative Tech. Workshop, St. Paul, MN, U.S.A.

Gilbert, B., Bettgenhaeuser, J., Upadhyaya, N., Soliveres, M., Singh, D., Park, R. F., Moscou, M. J., and Ayliffe, M. 2018. Components of Brachypodium distachyon resistance to nonadapted wheat stripe rust pathogens are simply inherited. PLoS Genet. 14:e1007636.

Haase, N. J., Beissinger, T., Hirsch, C. N., Vaillancourt, B., Deshpande, S., Barry, K., Buell, C. R., Kaeppler, S. M., and de Leon, N. 2015. Shared genomic regions between derivatives of a large segregating population of maize identified using bulked segregant analysis sequencing and traditional linkage analysis. G3: Genes Genomes Genet. 5:1593-1602.

Hall, A., Bastow, R. M., Davis, S. J., Hanano, S., McWatters, H. G., Hibberd, V., Doyle, M. R., Sung, S., Halliday, K. J., Amasino, R. M., and Millar, A. J. 2003. The TIME FOR COFFEE gene maintains the amplitude and timing of Arabidopsis circadian clocks. Plant Cell 15:2719-2729.

Heath, M. C. 2000. Nonhost resistance and nonspecific plant defenses. Curr. Opin. Plant Biol. 3:315-319.

Jin, Y., Szabo, L. J., Pretorius, Z. A., Singh, R. P., Ward, R., and Fetch, T., Jr. 2008. Detection of virulence to resistance gene $S r 24$ within race TTKS of Puccinia graminis f. sp. tritici. Plant Dis. 92:923-926.

Jin, Y., Szabo, L. J., Rouse, M. N., Fetch, T., Jr., Pretorius, Z. A., Wanyera, R., and Njau, P. 2009. Detection of virulence to resistance gene Sr36 within the TTKS race lineage of Puccinia graminis f. sp. tritici. Plant Dis. 93:367-370.

Jones, J. D. G., and Dangl, J. L. 2006. The plant immune system. Nature 444:323-329.

Kosugi, S., Momozawa, Y., Liu, X., Terao, C., Kubo, M., and Kamatani, Y. 2019. Comprehensive evaluation of structural variation detection algorithms for whole genome sequencing. Genome Biol. 20:117.

Langmead, B., and Salzberg, S. L. 2012. Fast gapped-read alignment with Bowtie 2. Nat. Methods 9:357-359.

Li, H. 2013. Aligning sequence reads, clone sequences and assembly contigs with BWA-MEM. arXiv. https://arxiv.org/pdf/1303.3997.pdf

Maluszynski, M., and Szarejko, I. 2005. Induced mutations in the green and gene revolutions. Pages 403-425 in: Proc. Int. Cong. "In the Wake of the Double Helix: from the Green Revolution to the Gene Revolution". R. Tuberosa, R. L. Phillips, and M. Gale, eds. Avenue Media, Bologna, Italy.

Martin, M. 2011. Cutadapt removes adapter sequences from highthroughput sequencing reads. EMBnet. J. 17:10-12.

Michelmore, R. W., Paran, I., and Kesseli, R. V. 1991. Identification of markers linked to disease-resistance genes by bulked segregant analysis: A rapid method to detect markers in specific genomic regions by using segregating populations. Proc. Natl. Acad. Sci. U.S.A. 88:9828-9832.

Mysore, K. S., and Ryu, C. M. 2004. Nonhost resistance: How much do we know? Trends Plant Sci. 9:97-104.

Nürnberger, T., Brunner, F., Kemmerling, B., and Piater, L. 2004. Innate immunity in plants and animals: Striking similarities and obvious differences. Immunol. Rev. 198:249-266.

Nürnberger, T., and Lipka, V. 2005. Non-host resistance in plants: New insights into an old phenomenon. Mol. Plant Pathol. 6:335-345.

Omidvar, V., Dugyala, S., Li, F., Rottschaefer, S. M., Miller, M. E., Ayliffe, M., Moscou, M. J., Kianian, S. F., and Figueroa, M. 2018. Detection of race-specific resistance against Puccinia coronata f. sp. avenae in Brachypodium species. Phytopathology 108:1443-1454.

Østergaard, L., and Yanofsky, M. F. 2004. Establishing gene function by mutagenesis in Arabidopsis thaliana. Plant J. 39:682-696.

Pabinger, S., Dander, A., Fischer, M., Snajder, R., Sperk, M., Efremova, M., Krabichler, B., Speicher, M. R., Zschocke, J., and Trajanoski, Z. 2014. A survey of tools for variant analysis of next-generation genome sequencing data. Brief. Bioinf. 15:256-278.

Panstruga, R., and Moscou, M. J. 2020. What is the molecular basis of nonhost resistance? Mol. Plant-Microbe Interact. 33:1253-1264.

Pretorius, Z. A., Singh, R. P., Wagoire, W. W., and Payne, T. S. 2000. Detection of virulence to wheat stem rust resistance gene $\mathrm{Sr} 31$ in Puccinia graminis f. sp. tritici in Uganda. Plant Dis. 84:203.
Rausch, T., Zichner, T., Schlattl, A., Stütz, A. M., Benes, V., and Korbel, J. O. 2012. DELLY: Structural variant discovery by integrated pairedend and split-read analysis. Bioinformatics 28:i333-i339.

Robert-Seilaniantz, A., Grant, M., and Jones, J. D. G. 2011. Hormone crosstalk in plant disease and defense: More than just jasmonatesalicylate antagonism. Annu. Rev. Phytopathol. 49:317-343.

Robinson, J. T., Thorvaldsdóttir, H., Winckler, W., Guttman, M., Lander, E. S., Getz, G., and Mesirov, J. P. 2011. Integrative genomics viewer. Nat. Biotechnol. 29:24-26.

Rotmistrovsky, K., Jang, W., and Schuler, G. D. 2004. A web server for performing electronic PCR. Nucleic Acids Res. 32:W108-W112.

Rutger, J. N. 1992. Impact of Mutation Breeding in Rice. Mutation Breeding Review, no. 8. International Atomic Energy Agency (IAEA), Vienna, Austria.

Sanchez-Villarreal, A., Shin, J., Bujdoso, N., Obata, T., Neumann, U. Du, S. X., Ding, Z., Davis, A. M., Shindo, T., Schmelzer, E., Sulpice, R., Nunes-Nesi, A., Stitt, M., Fernie, A. R., and Davis, S. J. 2013. TIME FOR COFFEE is an essential component in the maintenance of metabolic homeostasis in Arabidopsis thaliana. Plant J. 76:188-200.

Sato, Y., Morita, R., Katsuma, S., Nishimura, M., Tanaka, A., and Kusaba, M. 2009. Two short-chain dehydrogenase/reductases, NONYELLOW COLORING 1 and NYC1-LIKE, are required for chlorophyll $\mathrm{b}$ and light-harvesting complex II degradation during senescence in rice. Plant J. 57:120-131.

Schneeberger, K., Ossowski, S., Lanz, C., Juul, T., Petersen, A. H., Nielsen, K. L., Jørgensen, J. E., Weigel, D., and Andersen, S. U. 2009. SHOREmap: Simultaneous mapping and mutation identification by deep sequencing. Nat. Methods 6:550-551.

Schuler, G. D. 1997. Sequence mapping by electronic PCR. Genome Res. 7:541-550.

Schulze-Lefert, P., and Panstruga, R. 2011. A molecular evolutionary concept connecting nonhost resistance, pathogen host range, and pathogen speciation. Trends Plant Sci. 16:117-125.

Shin, J., Du, S., Bujdoso, N., Hu, Y., and Davis, S. J. 2013. Overexpression and loss-of-function at TIME FOR COFFEE results in similar phenotypes in diverse growth and physiological responses. J. Plant Biol. 56:152-159.

Shin, J., Heidrich, K., Sanchez-Villarreal, A., Parker, J. E., and Davis, S. J. 2012. TIME FOR COFFEE represses accumulation of the MYC2 transcription factor to provide time-of-day regulation of jasmonate signaling in Arabidopsis. Plant Cell 24:2470-2482.

Sikora, P., Chawade, A., Larsson, M., Olsson, J., and Olsson, O. 2011. Mutagenesis as a tool in plant genetics, functional genomics, and breeding. Int. J. Plant Genomics 2011:314829.

Singh, R. P., Hodson, D. P., Jin, Y., Lagudah, E. S., Ayliffe, M. A., Bhavani, S., Rouse, M. N., Pretorius, Z. A., Szabo, L. J., Huerta-Espino, J., Basnet, B. R., Lan, C., and Hovmøller, M. S. 2015. Emergence and spread of new races of wheat stem rust fungus: Continued threat to food security and prospects of genetic control. Phytopathology 105: 872-884.

Stakman, E. C., Stewart, D. M., and Loegering, W. Q. 1962. Identification of Physiologic Races of Puccinia graminis var. tritici. Publ E-617. United States Department of Agriculture-Agricultural Research Service, Washington, DC, U.S.A.

Thordal-Christensen, H. 2003. Fresh insights into processes of nonhost resistance. Curr. Opin. Plant Biol. 6:351-357.

Uma, B., Rani, T. S., and Podile, A. R. 2011. Warriors at the gate that never sleep: Non-host resistance in plants. J. Plant Physiol. 168:2141-2152.

Ye, K., Schulz, M. H., Long, Q., Apweiler, R., and Ning, Z. 2009. Pindel: A pattern growth approach to detect break points of large deletions and medium sized insertions from paired-end short reads. Bioinformatics 25:2865-2871.

Zambino, P. J., Kubelik, A. R., and Szabo, L. J. 2000. Gene action and linkage of avirulence genes to DNA markers in the rust fungus Puccinia graminis. Phytopathology 90:819-826.

Zhao, Y., Thilmony, R., Bender, C. L., Schaller, A., He, S. Y., and Howe, G. A. 2003. Virulence systems of Pseudomonas syringae pv. tomato promote bacterial speck disease in tomato by targeting the jasmonate signaling pathway. Plant J. 36:485-499.

Zhong, S., Leng, Y., Friesen, T. L., Faris, J. D., and Szabo, L. J. 2009. Development and characterization of expressed sequence tag-derived microsatellite markers for the wheat stem rust fungus Puccinia graminis f. sp. tritici. Phytopathology 99:282-289. 